\title{
Rapid turnover of actin in dendritic spines and its regulation by activity
}

Erin N. Star, David J. Kwiatkowski and Venkatesh N. Murthy

Nat. Neurosci. 5, 239-246 (2002)

Due to a printing error, the green in Fig. 2a was difficult to see. The corrected figure is reprinted below.

a

Stable

filaments

Dynamic

filaments
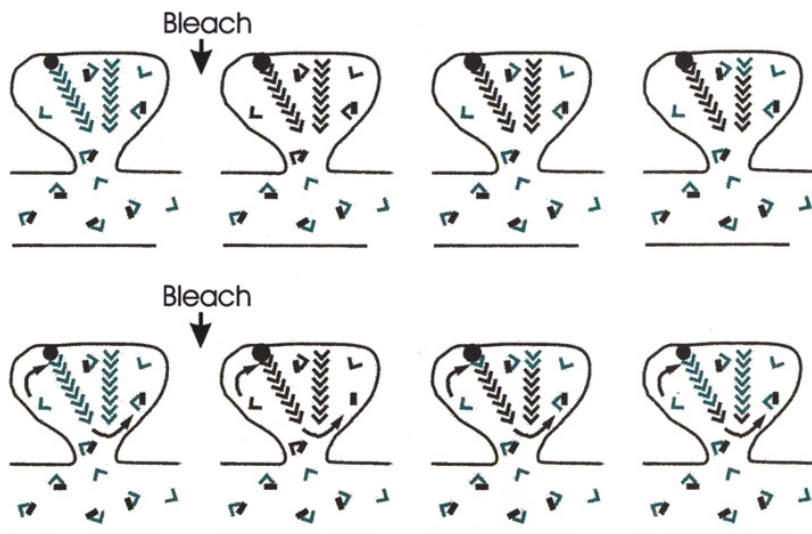

Bleach
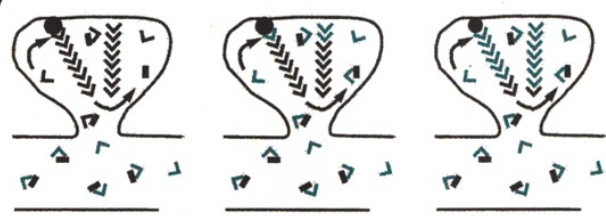

Fig. 2. (a) Illustration of the FRAP method. Fluorescent actin monomers (green barbs) and filaments are free or bound to mobile or immobile proteins (black rectangles and circles), and the binding affinities can be regulated. Selective photobleaching of actin in the spine is followed by rapid exchange of mobile monomers (free and bound) with the dendritic region and recovery of fluorescence. If actin filaments do not turn over, filamentous actin will remain nonfluorescent (top panels) and the spine will not recover its fluorescence. On the other hand, if filaments are turning over rapidly, fluorescent monomers will be continuously incorporated into them and the bleached actin monomers will be exchanged out of the spine. The spine will thus recover its fluorescence (bottom panels).

\section{Cooperation between independent hippocampal synapses is controlled by glutamate uptake}

Nina Arnth-Jensen, Denis Jabaudon and Massimo Scanziani

Nat. Neurosci. 5, 325-331(2002)

Due to a proof correction error, a sentence on page 329 beginning on line 19 was misprinted as "The amplitude-weighted average time course of the NMDAR-mediated EPSCs when the two pathways were simultaneously stimulated with four $100-\mathrm{Hz}$ stimuli every $6 \mathrm{~s}$ was slower than that of EPSCs evoked by independent stimulation of the two pathways $(8 \pm 1 \% ; P<0.0005 ; n=6)$." This sentence should have read, "NMDAR-mediated EPSCs evoked when the two pathways were simultaneously stimulated with four $100-\mathrm{Hz}$ stimuli every $6 \mathrm{~s}$ were slower than the amplitude-weighted average time course of EPSCs evoked by independent stimulation of the two pathways $(8 \pm 1 \% ; P<0.0005 ; n=6)$."

\section{corrigenda}

\section{Glial cells generate neurons: the role of the transcription factor Pax6}

Nico Heins, Paolo Malatesta, Francesco Cecconi, Masato Nakafuku, Kerry Lee Tucker, Michael A. Hack, Prisca Chapouton, Yves-Alain Barde and Magdalena Götz

Nat. Neurosci. 5, 308-315 (2002)

The authors wish to correct the affilitation of Yves-Alain Barde, which is ${ }^{4}$ Friedrich Miescher Institute for Biomedical Research, Basel, Switzerland. In addition, the size of the coding sequence of Pax6 inserted into the retroviral vector was incorrectly reported as $783 \mathrm{bp}$ on page 314 . The correct size is $1873 \mathrm{bp}$. 